\title{
«Die Kunst des Fragens» - Psychoanalyse an der Universität Zürich? Ein Gespräch mit Brigitte Boothe ${ }^{1}$
}

\author{
Laura Wolf (Zürich)
}

Zur Person: Prof. em. Dr. phil. Brigitte Boothe studierte zum einen Germanistik, Romanistik, Philosophie und erwarb zum anderen 1977 das Diplom in Psychologie. Sie dissertierte im selben Jahr in Philosophie über Wittgensteins Konzepte der Beschreibung und der Lebensform. Über Sprache und Psychoanalyse folgte 1988 die Habilitation an der Medizinischen Fakultät der Universität Düsseldorf. Von 1990 bis 2013 war sie Inhaberin des Lehrstuhls für Klinische Psychologie und stand der Abteilung Klinische Psychologie, Psychotherapie und Psychoanalyse am Psychologischen Institut der Universität Zürich (UZH) vor. In dem von ihr entwickelten qualitativen Forschungszugang (Erzählanalyse JAKOB) widmete sie sich klinischen Erzählungen und publizierte zur Psychoanalyse der Weiblichkeit, Kommunikation und Narration in der Psychotherapie wie zur Psychologie des Wünschens. Zudem führte sie eine universitäre postgraduale Weiterbildung in Psychoanalytischer Psychotherapie ein, welche seit ihrer Emeritierung an der UZH nicht mehr fortgeführt wird. Ihre Weiterbildung zur Psychoanalytikerin (DPG, $D G P T)$ absolvierte sie am freien Institut für Psychoanalyse und Psychotherapie Düsseldorf e.V. (IPD) und bildete sich zusätzlich in Gesprächspsychotherapie wie Psychodrama weiter. Derzeit arbeitet sie in freier Praxis in Zürich. Für ihr Lebenswerk ist Frau Boothe 2018 mit dem Preis der Dr. Margrit Egnér-Stiftung ausgezeichnet worden.

Schlüsselwörter: Universität, Institutionalisierung, Schweiz

Laura Wolf: Vor 30 Jahren haben Sie Ihren Lehrstuhl für Klinische Psychologie am Psychologischen Institut der Universität Zürich als Nachfolge von Prof. em. Ulrich Moser angetreten, standen 23 Jahre der Abteilung Klinische Psychologie, Psychotherapie und Psychoanalyse vor und sind nun selbst vor sieben Jahren aus der Hochschulinstitution ausgeschieden. Doch beschäftigen Sie sich 
weiterhin mit der Psychoanalyse, arbeiten als Psychoanalytikerin in eigener Praxis. Wie ist das für Sie, Psychoanalyse nun ohne Institution zu betreiben?

Brigitte Boothe: Auf das Ende meiner beruflichen Arbeitszeit mit der Emeritierung habe ich mich damals sehr gefreut. Ich stellte mir vor, dass jetzt noch ganz andere Dinge begännen, die zu kurz gekommen waren. Damit meinte ich gar nicht unbedingt Freizeitaktivitäten, sondern auch, Neues kennenzulernen, etwa in Wissensgebieten jenseits von Psychologie, Psychotherapie und Psychoanalyse. Überhaupt war ich neugierig, bestimmte Gewohnheiten einmal durch etwas ganz Anderes zu ersetzen. Das schwebte mir damals vor und ist auch noch nicht ganz verschwunden. Nur bin ich noch nicht so weit gekommen. (Lacht)

Wie die Hochschulen allgemein befindet sich auch die Universität Zürich in einem starken Veränderungsprozess, der insbesondere mit der Bologna-Reform zusammenhängt. Das betrifft beispielsweise die Universität als Organisationseinheit mit der sehr starken Ausdehnung im Bereich der Administration, sowohl auf den höchsten Führungsebenen wie auch in den Seminaren und Instituten. Auch die Organisation und Strukturierung der universitären Lehre ist davon betroffen und verlangt im verwickelten Prozess anhaltender Re- und Neuorganisation ein hohes Mass an Anpassung und Einpassung, dem sich Kollegen und Kolleginnen am Anfang und in der Mitte ihrer akademischen Laufbahn - im Vergleich zu mir - vermutlich mit grösserem Engagement stellen können. Mit grosser Dankbarkeit, Wertschätzung und Freude denke ich aber an die immer neue Bereitschaft, die kreative Bereitschaft, die Phantasie und die didaktischen Fähigkeiten der Mitarbeitenden, sich in der Lehre einzusetzen, Studierende für die Psychoanalyse zu begeistern und sie bei ihren Seminar- und Abschlussarbeiten zu begleiten.

Prof. Dr. Ulrich Moser verfügte bereits über eine mit dem Lehrstuhl verbundene Beratungsstelle, auch eine vorzüglich aufgebaute Einrichtung zur Herstellung und Dokumentation von Video- und Audioaufnahmen von Beratungsund Therapiegesprächen. Die Beratungsstelle konnte als psychotherapeutisches Angebot ausgebaut werden. So war es im Rahmen der von mir geleiteten Abteilung für klinische Psychologie, Psychotherapie und Psychoanalyse für die Mitarbeitenden in psychoanalytischerWeiterbildung und für mich selbst möglich, in einem bestimmten Umfang auch psychotherapeutische und psychoanalytische Praxis zu betreiben. Wir konnten Audio- und Videodokumentationen integrieren und erhielten auf diese Weise einen idealen Zugang für eine psychoanalytische wie psychotherapeutische Forschung mit den Schwerpunkten Kommunikations-, Prozess- und klinische Erzählanalyse. 
Als die Lehrstuhlinhaber Detlev von Uslar (Ordinarius für Allgemeine theoretische Psychologie), Norbert Bischof (Ordinarius für Allgemeine Psychologie experimentell-mathematischer Richtung; Anm. d. R.) und später auch Inge Strauch (Ordinaria für Klinische Psychologie; Anm. d. R.) sowie René Hirsig (Psychologische Methodenlehre) emeritierten, wurde das bis heute expandierende Psychologische Institut durch die Neubesetzungen stärker naturwissenschaftlich, experimentell ausgerichtet. Im Zentrum der klinischen Psychologie steht die verhaltenstherapeutische Orientierung (Prof. U. Ehlert, Prof. A. Maercker, Prof. G. Bodenman; Anm. d. R.).

Laura Wolf: Sie leiteten auch eine universitäre postgraduale Weiterbildung in Psychoanalytischer Psychotherapie, ähnlich zu den übrigen bereits erwähnten Klinisch-psychologischen Professor/innen am Institut, die ebenfalls ihre eigenen Weiterbildungen in ihren Schulausrichtungen installierten. Was war Ihre Motivation auch die Weiterbildung in psychoanalytischer Psychotherapie zu akademisieren? In Zürich gab es mit dem Freud-Institut (FIZ) und dem Psychoanalytischen Seminar Zürich (PSZ) bereits zwei Ausbildungsorte.

Brigitte Boothe: Zunächst begann es bei uns mit einzelnen Modulen, die später zu einem vierjährigen Gesamtprogramm, eben zur psychoanalytisch-psychotherapeutischen Weiterbildung weiterentwickelt wurden. Damals wurde an der UZH viel in Weiterbildungen investiert, und das entsprechende Angebot expandiert bis heute in allen Fakultäten. Wir erhielten die Chance durch den Master of Advanced Studies (MASP) in Postgradualer Weiterbildung in Psychoanalytischer Psychotherapie einen universitären Abschluss mit spezifisch beruflicher Qualifizierung anzubieten. Die Universität bot eine Anschubfinanzierung an und unterstützte die Institute und Lehrstühle. Meine eigene psychoanalytischeWeiterbildung in Düsseldorf fand an einem von Frau Prof. Dr. Annelise Heigl-Evers aufgebauten Institut der DGPT statt; es war eng mit ihrem Lehrstuhl an der Universität Düsseldorf verbunden und ausgezeichnet organisiert; wir Weiterbildungsteilnehmenden profitierten davon und konnten in nützlicher Frist zum Abschluss kommen.

Laura Wolf: Haben Sie denn von den hiesigen Instituten Reaktionen zu Ihrem Weiterbildungsmodell vernommen?

Brigitte Boothe: Vom Freud-Institut oder vom Psychoanalytischen Seminar Zürich? 
Laura Wolf: Ja.

Brigitte Boothe: Nicht direkt. Aber mit grossen Vorbehalten war in jedem Fall zu rechnen. Andererseits waren Psychoanalytiker/innen beider Institutionen (FIZ, PSZ) an unserem Weiterbildungsangebot als Dozierende, Supervidierende und Lehranalytiker/innen kontinuierlich beteiligt.

Laura Wolf: Inwiefern haben Sie von den sich in Weiterbildung Befindenden Rückmeldungen erhalten, weshalb sie sich für die Variante an der UZH entschieden haben und gegen die übrigen Institute?

Brigitte Boothe: Nicht in einerWeise, dass ich mich konkret erinnern könnte. Vielleicht hätte man das systematischer erfragen sollen. Aber ich glaube, für die Leute war es motivierend, dass sowohl die Kosten als auch die Ziele überschaubar waren. Man hatte eine übersichtliche Orientierung. Es gab Inhalte, die spezifisch mit unseren Forschungsschwerpunkten verbunden waren, wie Erzählanalyse, Arbeit mit Therapietranskripten, Traumanalyse, Kommunikationsanalyse, Arbeit mitVideoprotokollen, Vertrauensbildung, Kreditierung, um nur einiges zu nennen.

Laura Wolf: Wenn Sie die derzeitige Reform des Psychotherapeutengesetzes zur Direktausbildung an Universitäten in Deutschland mitverfolgt haben, so konnte man bspw. von Marianne Leuzinger-Bohleber Befürchtungen vernehmen, dass es dabei zu einer noch grösseren Marginalisierung der Psychoanalyse kommen könnte - eben durch die kaum noch vorhandene akademische Verankerung der Psychoanalyse und die parallele traditionell ausserakademische Vereinsstruktur. Sie sind andererseits gerade den Weg einer fortschreitenden Akademisierung mitgegangen, haben ihn selbst in Düsseldorf als förderlich erlebt. Doch ist auch Ihr Weiterbildungsmodell nicht in eine Dachgesellschaft wie bspw. der Internationalen Psychoanalytischen Vereinigung (IPV) integriert gewesen - stärker psychoanalytisch institutionalisiert. Was waren Ihre Beweggründe?

Brigitte Boothe: Die IPV ist zweifellos ein imposantes, vielfältiges, facettenreiches Gebäude. Es gibt dort extrem viel Initiative, äusserst interessante Aktivitäten, Forschungsforen, hochinteressante Persönlichkeiten, kulturwissenschaftliche Beteiligungen, Mitarbeit an den neuen diagnostischen Systemen usw. Doch wenn ich daran denke, mit welch extremen Hürden Prof. Dr. Jürgen Körner auf seinem Weg zur Anerkennung der Deutschen Psychoanalytischen Gesellschaft 
(DPG) durch die IPV konfrontiert war, gibt es Anlass zur Skepsis. Ein extremer Anspruch an Dominanz und Führung ist bei der IPV vorhanden - allein schon diese Vierstundendiskussion - ein bemerkenswertes Ausmass an Normativität, u. a. im Blick auf Traditionsbindung. Tradierte Grundfesten sind aber nicht immer rational vertretbar oder argumentativ ausreichend stichhaltig. Problematisch ist der Führungsanspruch, wer über die wahre Psychoanalyse bestimmt.

Laura Wolf: Sie meinen einen Hang zum Dogmatismus ...

Brigitte Boothe: Ja, das würde ich schon sagen.

Laura Wolf: Das ist ja etwas, womit die Psychoanalyse per se stark verbunden wird, dogmatisch zu wirken. Wie war das für Sie 23 Jahre die Psychoanalyse an der Philosophischen Fakultät einer Universität zu vertreten?

Brigitte Boothe: Ich will das bezugnehmend auf das Stichwort «Dogmatismus» und meine Weiterbildungserfahrung Ende der 70er/Anfang der 80er Jahre am Düsseldorfer Institut unter der Leitung von Frau Prof. Heigl-Evers erläutern. Ich glaube, dass es gut war, dass sich jenes Institut zunächst ausserhalb einer Dachvereinigung gegründet und entwickelt hat. Es war verbunden mit dem Lehrstuhl Heigl-Evers' für Psychotherapie und Psychosomatik an der Universität Düsseldorf, und als gleichzeitige ärztliche Klinikdirektorin leitete sie eine psychosomatische Abteilung mit einer Ambulanz, einer Klinik sowie einer Tagesklinik. So kam man rasch in Kontakt mit Patient/innen, beispielsweise im Konsiliardienst für die Organmedizin o. Ä. Dadurch war man schnell mit Formen psychischen und physischen Leidens konfrontiert, die neue und flexible Diagnoseund Indikationsstellungen verlangten. Behandlungsangebote wie eine klassische Vierstundenanalyse waren da kaum umsetzbar. Ich erinnere mich noch gut, wie ich - inspiriert von Hermann Argelanders «Das Erstgespräch in der Psychotherapie»; ohne Zweifel bis heute ein schönes und zu recht vielgelesene Buch - versuchte, ein psychoanalytisches Erstgespräch zu führen, also auf Emotionen, persönliches Erleben, Beziehungen zu fokussieren. Und der Patient entgegnete entrüstet: «Wo bin ich hier eigentlich? Das geht Sie erstens gar nichts an und zweitens habe ich Bauchschmerzen!» (Lacht)

Psychosomatische Patient/innen und Personen mit komplexen Störungsbildern oder in psychosozialen Krisen waren für mich eine grosse Herausforderung; letztlich fand ich diese Auseinandersetzung ungemein hilfreich: Was mache ich 
jetzt konkret in so einer Gesprächssituation? Wie bringe ich das mit den gelernten Konzepten in Verbindung? Heute erkenne ich sehr deutlich, dass Frau Heigl-Evers eine Pionierin in der Behandlung insbesondere psychosomatischer Patient/innen, Persönlichkeiten mit Abhängigkeitserkrankungen und Entwicklungsstörungen war. Annelise Heigl-Evers und Franz Heigl legten differenzierte interaktionell ausgerichtete Behandlungsangebote für diese schwierigen Patient/innen vor, die auch heute in derWeiterbildung gelehrt werden. Wir Weiterbildungsteilnehmende hatten das Glück, vor Ort etwas Neues zu erproben; und wir erfuhren, wie wichtig und bedeutsam es ist, die kommunikative Situation im Hier und Jetzt des therapeutischen Gesprächs zu beachten und auf professionelle Art wirksam werden zu lassen. Das hat mich langfristig ermutigt, Kommunikation, und Erzählen als Forschungsgegenstände zu vertiefen. Doch ging es sehr wohl auch oder gerade damals - innerhalb und ausserhalb der Weiterbildung - immer wieder um die leidige Frage Wer von uns ist jetzt eigentlich der oder die wahre Psychoanalytiker/ in?, mit den typischen Grabenkämpfen auf dieser Ebene oder: Ist das noch Psychoanalyse, was Ihr hier macht?

Kernberg und viele andere haben bekanntlich über Probleme in Ausbildungsinstituten geschrieben. Sie thematisieren die Tendenz zum Normativismus oder auch fehlende intellektuelle Offenheit. Vermutlich sind - gruppenpsychoanalytische Forschung belegt das; übrigens sind Heigl-Evers und Heigl auch hier Pioniere - Psychoanalytiker/innen nicht die einzigen, die in eine Dynamik der Inklusion und Exklusion gelangen. Freud war hier ein fragwürdiges Vorbild, trotz seines mutmachenden Diktums, das es in aller Radikalität darum gehe, Denkverbote aufzuheben.

Daher fand ich eine Institution wie die Philosophische Fakultät ganz schön. Ich hatte vor der Psychologie eben noch Geisteswissenschaften studiert, sodass es grossartig war, dort im Kontakt zu sein. Ich habe es von vornherein spannend gefunden, sich interdisziplinär austauschen zu können. Und in meinem speziellen Fall spielte die Erzählforschung eine besondere Rolle.

Laura Wolf: Interessanterweise ist das Psychologische Institut der UZH entgegen seiner zunehmenden naturwissenschaftlichen Ausrichtung weiterhin Teil der Philosophischen Fakultät und nicht etwa einer naturwissenschaftlichen Fakultät wie an anderen Hochschulen. Man könnte meinen, die hier betriebene Psychologie bewege sich von einem Austausch mit den übrigen Fakultätsfächern weg. Doch auch bei der ausserakademischen psychoanalytischen Vereinsstruktur fällt ein Mechanismus des Ausschlusses, der Abschottung auf, 
wie Sie ihn zuvor beschrieben haben. Alfred Lorenzer schrieb einmal, dass die Universitätspsychoanalyse insbesondere in der Auseinandersetzung mit den benachbarten wissenschaftlichen Disziplinen privilegiert und gefordert sei. Hatten Sie somit eine besondere Aufgabe inne?

Brigitte Boothe: Nein, das könnte ich jetzt nicht sagen. Wir haben 2005 an der UZH und der ETH Zürich ein Interdisziplinäres Psychoanalytisches Forum (IPF) gegründet, das bis zum Ende meiner Amtszeit initiativ war. Dazu gehörten z. B. Prof. Dr. Elisabeth Bronfen (Englisches Seminar, UZH; Anm. d. R.), Prof. em. Dr. Reinhard Fatke (Institut für Erziehungswissenschaft, UZH; Anm.d. R.), Prof. em. Dr. Georg Kohler (Philosophisches Seminar, UZH; Anm. d. R.), Prof. Dr. Philipp Stoellger von der Theologischen Fakultät der UZH, heute Professor in Heidelberg, und Prof. Dr. Michael Hampe seitens der ETH Zürich. Das war ein Schritt die Psychoanalyse auch auf interdisziplinärer Ebene als Diskussionsforum und Debattenkultur anzubieten. Immerhin organisierten wir Veranstaltungen; und u. a. erschienen zwei Bücher (s. Literatur; Anmd. d. R.). Es waren eine kulturwissenschaftliche, theologische, philosophische und sozialwissenschaftliche Öffnung damit verbunden.

Laura Wolf: Und im Rahmen des Psychologischen Instituts, inwiefern kam Ihnen eine besondere Rolle zu, oder waren Sie einfach eine von vier ordentlichen klinisch-psychologischen Professor/innen?

Brigitte Boothe: Insbesondere in der ersten Zeit, als eine Kultur der wechselseitigen Individualitätspflege bestand, man könnte auch von einer Art Alleinstellungspflege im Kleinen sprechen; ich denke an die Kollegin und die Kollegen Rainer Hornung, Heinz Gutscher, Norbert Bischof, Inge Strauch, Detlev von Uslar, René Hirsig und François Stoll - da war das eigentlich ein wechselseitiger Austausch bzw. eine wechselseitige freundliche Distanz. Jede/r positionierte sich mit dem eigenen Profil und im eigenen Terrain.

Später entfaltete sich eine Kollektivität übergeordneten gemeinsamen Positionsbezugs. Die klinische Psychologie psychoanalytischer Orientierung war dort nicht mehr systematisch integriert. So wie das wohl auch für die Psychoanalyse an der Universität gefürchtet wird - wenn man die Literatur darüber liest -, dass es keine Nachbesetzungen der Lehrstühle mit Psychoanalytiker/innen gibt u. Ä.

Andererseits meine ich, dass sich derzeit auch viele weibliche Repräsentanten für die Psychoanalyse im Hochschulraum einsetzen: Prof. Dr. Vera King in Frankfurt oder Prof. Dr. Ilka Quindeau als Präsidentin der International Psycho- 
analytic University (IPU) in Berlin. Sie erwähnten bereits die wissenschaftliche vielfach präsente, sehr engagierte und erfolgreiche Marianne Leuzinger-Bohleber für eine ältere Generation. Doch auch jüngere Personen wie Prof. Dr. Svenja Taubner von der Universität Heidelberg hat bereits an mehreren Lehrstühlen erfolgreich gewirkt. Sie treibt das Konzept der Mentalisierung stark voran und auch ihre Nachfolgerin in Klagenfurt, Univ.-Prof. Dr. Sylke Andreas, setzt dazu die Arbeiten fort. Was da geleistet wird, scheint mir doch sehr aktiv und produktiv zu sein - aber nicht nur in der klinischen Psychologie, sondern auch in der Sozialpsychologie und Kulturwissenschaft.

Laura Wolf: In Zürich ist das vielleicht ein beispielhafter Prozess: Mit dem Generationenwechsel insbesondere auf den klinischen Professuren bzw. deren Ausbau von 1999 an, scheint sich ein Shift in der akademischen Psychologie bemerkbar zu machen, eine starke Ausrichtung auf Konzepte der Neuro- und Kognitionspsychologie, Anlehnungen an eineVerhaltensbiologie. Gleichzeitig wächst das Institut enorm. Mit 16 ordentlichen Professuren und ca. 13 weiteren Professuren u. a. aus Förderungsstellen des Schweizerischen Nationalfonds (SNF), Assistenzen mit Tenure Track, ausserordentlicher Ernennung oder ad personam wie unglaublichen Studierendenzahlen ist es heute das vielleicht grösste psychologische Institut im deutschsprachigen Raum. Und doch scheint es für eine/n Professor/in mit psychoanalytischer Ausrichtung keinen Platz zu geben, obgleich sich ein psychoanalytisches Theorieninteresse in der Studierendengruppe abbildet. Auch ist mir derzeit an der Universität Zürich gesamthaft kein Lehrstuhl mit psychoanalytischer Denomination bekannt; vielleicht gibt es aber auf längere Sicht neue Aussichten und psychoanalysefreundliche Perspektiven.

Sie hatten einmal den Neuropsychologen Mark Solms als Redner in das Gästekolloquium des Psychologischen Instituts eingeladen. In einem Buchbeitrag zu «Psychoanalyse und Naturwissenschaft» (2017) vertritt er die Ansicht, die akademische Psychologie schliesse die Seele aus, da sie lediglich die objektiven Manifestationen der Seele untersuche und dadurch die Seele als Subjektives aussen vorlasse. Gemeinsam mit der Psychiatrie, die zu einer Psychopharmakologie werdend die Seele in objektive Neurotransmitter transformiere, bildeten sie, von ihm zugespitzt formuliert, «Abwehrstrukturen gegen die Psychoanalyse». Was denken Sie zum Verhältnis akademischer Psychologie und Psychoanalyse in einer universitären Einrichtung? 
Brigitte Boothe: (Denkt lange nach) Ja, was ist eigentlich akademische Psychologie? Nun, da fange ich bei der Seele an und komme später zum akademischen Teil zurück, weil ich das für eine wichtige Frage halte.

Die Seele hat in der Jungschen Tiefenpsychologie ihren systematischen Platz. Für die Jungianer wird die Idee der Seele und des Seelischen in Forschung und Praxis breit und differenziert behandelt - bei Freud hingegen überhaupt nicht. Freud hat die Konzeption eines psychischen Apparats vorgestellt. Es gibt einen Apparat und ein Lustprinzip. Wenn er von so etwas wie von illusionistischen Rationalisierungen spricht, wird sehr deutlich, mit welcher offensiven Skepsis er der «Seele» begegnet und wie wenig er den Vorstellungen vom «Seelischen» zutraut, dass auf ihrer Basis theoretisch und empirisch etwas Fruchtbares entwickelt werden könnte. Selbst die Liebe, genauer die Verliebtheit, lässt er schrumpfen auf eine illusionistische Verblendung; sie sei nichts weiter als eine «Überschätzung des Sexualobjekts». Die Psychoanalyse hatte, was die Seele anbelangt, zumindest in der älteren psychoanalytischen Tradition, eher einen skeptischen Standpunkt. Auch die nachfolgenden Theorieausrichtungen der Ichpsychologie, der Objektbeziehungspsychologie, im weiteren Verlauf des relationalen Turn, der Säuglingsforschung - erneut Stichwort Mentalisierung - findet man, meines Wissens, keine systematische Anstrengung, ein Seelenkonzept neu zu fassen oder zu integrieren.

\section{Laura Wolf: Sondern?}

Brigitte Boothe: Eben, das frage ich mich auch. Was ist nun eigentlich der Gegenstand der Psychoanalyse? Wie ist der Gegenstand zu konstituieren? Ich weiss es nicht. Ich könnte Ihnen keine Antwort geben, wenn ich genau hinschaue. Ist es die Mechanik des Lebens, um die es geht? Ist es letztlich das Darwin'sche Überleben, bereichert um das Lustprinzip? Vielleicht genauer gefasst und hier berührt es meine Faszination, die Konzeptualisierung des Wünschens? Was in der Idee des Wünschens steckt, wie man sozusagen mit dem Erwerb des Bewusstseins sein Befinden regulieren kann, das finde ich enorm spannend.

Laura Wolf: Das wäre dann die seelische Aktivität schlechthin.

Brigitte Boothe: Aber da kann doch ein Jungianer nur lachen. Das soll die Seele sein - so ein Krümelchen? (Beide lachen) 
Nun gut, ich weiss nicht, was die Psychoanalyse da eigentlich gemacht hat. Ich bin deutlich gegen ihre normativen Implikationen. Ich bin auch gegen dieses Guruhafte. Freud wurde durchaus immer wieder und wird es gelegentlich auch heute zum Guru oder als «Meisterdenker», stilisiert - gerade auch in den publizistischen Medien. Aber wenn die Autorität in der Wissenschaft nicht die Person sein soll, sondern die Sache, dann ist da etwas in Schieflage geraten. Das weckt berechtigtes Misstrauen. Andererseits stand Freud schon zu seinen Lebzeiten in der Kritik. Selbst seinen Anhängern war seine Traumtheorie nicht genehm, die Weiblichkeitstheorie schon mal gar nicht, und dass er «seinen Neurotica» nicht mehr «glauben» wollte, wurde zum Skandal. Jeffrey Masson, der ein glühender Analytiker war und in der Szene als Wunderkind galt, durchforstete die Archive und fand schliesslich, Freud habe die Kinder an die Gesellschaft verraten. Mit seinen Thesen in «Was hat man dir, du armes Kind getan?» löste er eine grosse Kontroverse aus und verlor seine Mitgliedschaft in den Berufsvereinigungen bzw. brach mit der Community. Angesichts der Buntheit der Konzepte verwundert es nicht, dass es auch innerhalb der Psychoanalyse nicht nur wissenschaftliche Auseinandersetzungen gibt, sondern auch Fehden, Aus- bzw. Eingrenzungen, moralische Verdächtigungen.

Laura Wolf: Wenn es schwierig ist, den Gegenstand der Psychoanalyse zu bestimmen, liesse sich denn etwas über den Gegenstand der Psychologie aussagen?

Brigitte Boothe: Ja, das führt zu weiteren Fragen wie: Was ist eigentlich das Wesen der Universität? Warum ist es attraktiv, an der Universität zu sein? Peter Schneider (Psychoanalytiker und PD an der UZH; Anm.d. R.) oder der bereits erwähnte Michael Hampe diskutieren dazu Ludwik Fleck und auch mir gefällt sehr, was er schreibt. ${ }^{2}$ Man könnte aber auch Bourdieu heranziehen, einen Praxistheoretiker, um unter einem soziologischen Gesichtspunkt zu betrachten, was wir als rationales Denken verstehen oder was wir als Errungenschaften des Wissens verstehen. Was macht eine Denkgemeinschaft aus? Was gibt es dabei für Kriterien? Was gibt es für Normen, die uns zusammenhalten? Das ist nicht die einfache Suche nach Wahrheit, sondern es wird auch ein kollektiver Identitätsausweis angestrebt. Das will die Universität ebenfalls - einen kollektiven Identitätsausweis repräsentieren. Und vielleicht würde sie immer noch sagen, dass es ihr um Erkenntnis gehe. Was ist der Weg dahin? 
Es müsste eigentlich seit Wilhelm von Ockham (mittelalterlicher Philosoph und Theologe; Anm. d. R.) ein demokratischerWeg sein. Jeder muss dieWerkzeuge haben, um selbst prüfen zu können, ob die Aussage Hand und Fuss hat. Das ist etwas, was die Wissenschaft von der Religion unterscheidet. Wissenschaft will, dass jeder, der die Werkzeuge benutzt, sagen kann: «Was Du da behauptest, hat Hand und Fuss». So können wir Aussagen über die Klimaveränderung mitvollziehen. Wir können diskutieren, was fake ist und was nicht. Und ich würde immer noch sagen, dass das eine gute Sache ist. Nur, erfüllt die Psychoanalyse dies?

Laura Wolf: Diese Frage hätte ich jetzt gerne Ihnen gestellt. Stattdessen wage ich mal die Überlegung, dass es vielleicht gerade das Werkzeug der psychoanalytischen Methode ist, die die Akzeptanz wie die Einbindung in das Spiel mit anderen wissenschaftlichen Disziplinen so schwierig macht. Denn die Zugänglichkeit zu dieser Methode ist mit einer Erkundung der eigenen (vermittelten) Subjektivität verbunden und einer fortwährenden Reflexion derVerwobenheit von Forschendem und zu Erforschendem. In meiner Unkenntnis beispielsweise der Physik kann ich das schlecht beurteilen, aber ich würde annehmen, dass dort eine grössere Distanz in der Methode gesucht wird, sich die Dinge vom Leib zu halten, vermeintlich «objektiv» zu bleiben. In der Psychoanalyse wäre solch ein Vorgehen dem Erkenntnisprozess hinderlich.

Brigitte Boothe: Obgleich ich es etwas leid bin, kehre ich doch nochmals zu Freud zurück. Als er begonnen hat, sich mit seinen Patient/innen zu befassen, bemerkte er deren jeweils ganz persönliche Welt. Bei den Psychotiker/innen sowieso, aber auch bei den damaligen Hysteriker/innen wurde ihre Verstrickung in teils unbewusste Dramen für dieWirklichkeit gehalten. Freud schloss, dass wenn er es fertigbrächte, mit den Patient/innen so zu arbeiten, dass sie den Ursprung ihrer Imaginationen, ihrer Wünsche und Ängste erkannten, dann begänne nach dem neurotischen Elend die Misere des Alltags. Das halte ich tatsächlich für eine ziemlich interessante Idee. Sie bedeutet extrem vereinfacht und auch schief: «Arbeite mit der Patientin oder dem Patienten so, dass ihre oder seine ganz persönliche individuelle Welt Ausdruck findet. Dass du ihr/ihm zuhörst in den von ihr/ihm erzählten Geschichten, in den Übertragungsprozessen und dann gehe zu einer neuen Perspektive über, wo man gemeinsam reflexiv auf das Ganze schaut.» Dieses reflexive Schauen wäre dann der Blick auf die Wirklichkeit. Das heisst, es kommt zu einer Verstrickung in der subjektiven Welt, doch wenn man darin bleibt, verharrt man in der Folie à deux, weshalb die Reflexion so betont wird. Also technisch 
gesprochen: einerseits Übertragung anwachsen lassen, andererseits gemeinsame Exploration, Reflexion, Urteilsbildung. So wechselt man zwischen einer zentrierten - «das bin jetzt ich» - und einer dezentrierten Perspektive: «Jetzt schauen wir mal ganz nüchtern, was ist hier eigentlich los.» Ein Erstsemester in der Medizin wird wahrscheinlich vor der Wunde erschrecken und dann über viele Jahre eine emotionale Distanz lernen, um zu fragen: «Mit was haben wir es hier zu tun?» Das fragt sich eine Analytikerin - ein entsprechendes Diktum von Morgenthaler ist mir hier sehr vertraut - auch, wenn der Patient jetzt gerade ihr diese Geschichte präsentiert. Dazu fallen ihr, auch theoretisch, konzeptuell, fallanalytisch inspiriert, diverse mögliche Antworten ein, z. B.: Das liege daran, dass sich das Unbewusste angesichts bestimmter Kindheitsereignisse, die sich aufgrund des psychischen Apparats - und heute würde man aufgrund verinnerlichter Objektbeziehungen sagen - entwickelt haben und so weiter. Das heisst, an der Wirklichkeit ist die Psychoanalyse sehr wohl interessiert. Trotzdem finde ich, dass Adolf Grünbaum (US-amerikanischer Physiker, Philosoph und Wissenschaftstheoretiker; Anm.d. R.) in Bezug auf Freud - und auf Anderes wage ich das nicht anzuwenden - einen Punkt getroffen hat. Man könne in einer Analyse, wenn man unterscheiden kann zwischen Gefälligkeitsantworten bzw. sogar -träumen und dem, was sich wirklich in der Beziehung bewege, zu einem Punkt gelangen, an dem man doch eine Erkenntnis über sich gewinnt, aber dieser Punkt sei nie sicher auszumachen.

Laura Wolf: Der Punkt der Erkenntnis.

Brigitte Boothe: Ja, das wäre in gewisser Weise ein qualitativer Sprung.

Laura Wolf: Nun machen wir uns gerade viele Gedanken über den Gegenstand der Psychoanalyse, aber auch zu selbstreflexiven Denkprozessen und ich frage mich, inwiefern es dafür noch ein Gefäss an der Universität Zürich, am Psychologischen Institut gibt. Etwas zugespitzt formuliert, taucht Subjektivität im Psychologiestudium lediglich als Störvariabele, integriert in eine Fehlerkalkulation im quantitativen Forschungsdesign auf.

Wie glauben Sie, finden heute Studierende den Weg zur Psychoanalyse und später in eine psychoanalytische Weiterbildung in Anbetracht der eher als Auskehren wahrnehmbaren Entwicklung an den Hochschulen? 
Brigitte Boothe: Da gibt es studentische Initiativen, wie das «Studentische Forum für Psychoanalyse an der UZH» (SFPa-UZH) und in Deutschland die «Interessengemeinschaft der Psychoanalyse an Universitäten» (IDPAU). In Köln ist sogar eine neue Professur für Klinische Psychologie und empirisch-quantitative Tiefenpsychologie soeben ausgeschrieben gewesen. Das lässt doch wieder hoffen. Und bei Interesse gibt es ja ein vielfältiges Angebot an Tagungen, Seminaren, Workshops in privaten Instituten und anderswo, umfangreiche Lektüre und sicherlich viel online zu finden.

Laura Wolf: Das setzt voraus, dass man bei sich ein Interesse verspürt und dann um die Mittel wie ihren Einsatz weiss, dem Interesse auch nachzuspüren. Meines Erachtens ist dabei jedoch die Rolle des Vermittlers nicht ausser Acht zu lassen. Sie haben selbst ein paarmal Frau Heigl-Evers erwähnt. Hat es nicht auch etwas damit zu tun, welchen Personen man, ob in jungen oder älteren Jahren, begegnet, welche man zum Vorbild wählt oder inspirierend findet? So wäre es eigentlich wichtig, dass mehr Menschen mit psychoanalytischem Hintergrund an den Universitäten vertreten wären.

Brigitte Boothe: Ja, es ist eine relevante Frage, wie Nachwuchs interessiert werden kann. Das wäre ja die Zukunft. Einerseits müssten die Institute mehr Zugänge schaffen, vielleicht auch über Medienkanäle, die näher zu jungen Leuten führen - Vorträge aufnehmen oder dergleichen. Doch braucht es auch Menschen, die sich innerhalb der Hochschulinstitutionen engagieren. Es ist von Vorteil, wenn das jemand ist, der oder die einen mitreissen kann. Jemand, der oder die eine Vorbildfunktion übernehmen möchte. Jemand, der auf wissenschaftlicher Ebene Fragen stellen kann, die zur Forschung motivieren, nicht nur zur eigenen Analyse, sondern zu so etwas wie: «Ich möchte weiterkommen. Ich möchte über Dinge genauer und vertiefter nachdenken können. Ich möchte das auch anderen beibringen können.» Dazu ist eine Universität oder auch eine Fachhochschule - für die Zürcher Hochschule für Angewandte Wissenschaften (ZHAW) ist bspw. Prof. Agnes von Wyl zu nennen -, ein Forschungszentrum wie auch eine Privatuniversität sicher geeignet.

Laura Wolf: Inwiefern braucht Ihrer Meinung nach überhaupt die Hochschulinstitution die Psychoanalyse und/oder die Psychoanalyse die Hochschulinstitution? 
Brigitte Boothe: Die Psychoanalyse verfügt einfach über ein viel grösseres Potenzial, als nur eine Therapieform zu sein. Sie eröffnet eindrucksvoll diverse Möglichkeitsräume und kann in vielen Wissenschaften und Anwendungen einen Beitrag leisten. Beispielsweise ist sie längst in der Arbeits- und Organisationspsychologie angekommen, im Beratungssektor präsent, hat Eingang in die Erziehungswissenschaften gefunden. Auch Förderungsnetzwerke wollen ihre Nachwuchsförderung um ein Mentoring ausbauen, weil eine viel umfassendere Lebensplanung dazugehört. Nicht nur: Wie kriegst Du eine gute Doktoratsbetreuerin oder einen Doktoratsbetreuer, sondern auch wie verbindest Du eigentlich Beruf und Privatleben?

Die Psychoanalyse war auch immer schon Kulturwissenschaft. Gesellschaftlich hat sie in Bezug auf Migration, Trauma und Gewalt früh bedeutende Erkenntnisse geliefert und fortwährend weiterentwickelt. Man denke an Luise Reddemann und ihre Leistung für Traumabehandlungen.

Ich glaube, wovon die Psychoanalyse und umgekehrt die Universität profitieren könnte, wäre mehr Feldkompetenz. Nehmen wir einen Begriff wie Narzissmus. Diagnostische Etiketten werden oft wie die Merkmale von Personen behandelt. Da bin ich nicht die einzige, die das sagt. Es sollte viel mehr Sorgfalt und Aufmerksamkeit darauf gerichtet werden, wie die Lebensverhältnisse der Personen sind. Eine Person ist beispielsweise ambitioniert und will Karriere machen. Wenn diese Person nicht ein Profilierungsinteresse hat und auch fähig ist, sich im sozialen Feld erfolgreich selbst darzustellen, wird sie nicht reüssieren. Also das Verhältnis von Pathologie und Lebensform - das scheint mir etwas zu sein, was mehr Berücksichtigung finden sollte.

Laura Wolf: Der Germanist Prof. Peter-André Alt hat zur Zeit seiner Präsidentschaft der Freien Universität Berlin in einer Rede die Psychoanalyse als die modernste aller Humanwissenschaften bezeichnet. Sie sprechen ebenfalls von ihrem Potenzial und doch sehen wir eine institutionelle Entwicklung des Auskehrens an den Universitäten und eine Einkehr in Privathochschulen. So haben sich mit der Gründung der IPU in Berlin, dem Expandieren der Sigmund Freud PrivatUniversität Wien (SFU) nach Paris, Ljubljana, Berlin, Mailand und Linz in den vergangenen ca. 15 Jahren private akademische Institutionen gebildet, die bspw. nach Aussage der IPU eine Lücke in der einseitigen naturwissenschaftlichen Ausrichtung der akademischen Psychologie schliessen wollen. Ist das derWeg die Psychoanalyse in der Hochschullandschaft überhaupt noch auf der Karte zu halten? Isoliert sie sich dadurch oder besammelt sie sich? 
Brigitte Boothe: Da wage ich keine Prognose. Die Psychoanalyse ist mit ihren vielen Anregungen und Initiativen so präsent, dass ich jetzt eigentlich nicht ihr Ende sehe. Manchmal, wenn man die Literatur zur Situation an den Universitäten liest, kann man schon einen beleidigten Ton wahrnehmen: «Wieso mögen die uns nicht?» Oder grandios gewendet: «Ist es ein herausragendes Merkmal, dass sie uns nicht sehen? Ist das gerade unser Ehrenzeichen?»

Bezüglich der IPU Berlin gefällt mir besonders, dass da viel - nicht, dass ich das Wort oft benutze - «Frauenpower» drin ist. Es war Frau Rohde-Dachser, die mit Jürgen Körner die Hochschule gegründet hat und es entwickelt sich sehr gut. Auch jetzt ist viel Fraueninitiative dabei, das ist ermutigend. Es werden interessante Leute gewonnen, relevante, aufregende, zukunftsfähige Projekte entwickelt. Da ist viel Originelles, und sie müssen sich nicht rumschlagen mit einer bremsenden Umgebung. Es kann also sein, dass das, was dort entsteht, hohen Anregungswert hat und nicht abgetan wird im Sinne von: «Also gut, dann lassen wir die Spinner machen.» Ausserdem, warum soll man nicht ein bisschen spinnen? Wir hatten immerhin auch Paul Feyerabend an der ETH Zürich. Und ist das Spinnen nicht ein kreatives Spezialgebiet der Psychoanalyse? Nochmals: «Kein Denkverbot»- das war und bleibt eine grossartige Losung.

Laura Wolf: Also die Universitäten könnten die Spinner wieder zulassen oder anders ausgedrückt ein (gesellschaftlicher) Ort sein, frei zu denken, wie das als Ideal im Setting von Couch und Sessel angenommen wird?

Brigitte Boothe: Ich habe vor kurzem ein kleines Textchen über die Psychoanalyse als die Kunst des Fragens gelesen. Die Kunst des Fragens klingt für mich toll - nicht dass man $z u$-gedeutet wird, sondern dass die eigenen Fragen etwas bewegen, etwas öffnen. Das kann Psychoanalyse. Die verrücktesten Sachen kann man da fragen.

Laura Wolf: Und es wäre schön, dies auch innerhalb von Hochschulwänden zu tun. Ich danke für das Gespräch!

\section{Literatur}

Argelander, H. ([1970] 2009). Das Erstinterview in der Psychotherapie. 8. unveränd. Auflage. Darmstadt: WBG.

Boothe, B. (Hrsg.). (2008). Ordnung und Ausser-Ordnung. Zwischen Erhalt und tödlicher Bürde. Bern: Huber. 
Boothe, B. (Hrsg.). (2013). Wenn doch nur - ach hätt ich bloß. Die Anatomie des Wunsches. Zürich: Rüffer \& Rub.

Boothe, B., Cremonini, S. \& Kohler, G. (Hrsg.). (2012). Psychische Regulierung, kollektive Praxis und der Raum der Gründe. Ein Problemaufriss. Würzburg: Königshausen \& Neumann.

Boothe, B. \& Frick, E. (2017). Spiritual Care. Zürich: Orell Füssli.

Boothe, B. \& Hermann, M.-L. (Hrsg.). (2018). Nachträglichkeit und Neubeginn. Themenheft der Zeitschrift Psychosozial 41, Nr. 154, Heft IV.

Fleck, L. (1980). Entstehung und Entwicklung einer wissenschaftlichen Tatsache. Einführung in die Lehre vom Denkstil und Denkkollektiv. Frankfurt a. M.: Suhrkamp.

Kernberg, O. F. (1986). Institutional Problems of Psychoanalytic Education. Journal of the American Psychoanalytic Association, 34, 43-59.

Kernberg, O. F. (1998). Dreißig Methoden zur Unterdrückung der Kreativität von Kandidaten der Psychoanalyse. Psyche, 52(3), 199-213.

Lorenzer, A. (1986). Tiefenhermeneutische Kulturanalyse. In Ders. (Hrsg.), Psychoanalytische Studien zur Kultur (S. 11-98). Frankfurt a. M.: Fischer Verlag.

Masson, J. M. (1984). Was hat man dir, du armes Kind, getan? Sigmund Freuds Unterdrückung der Verführungstheorie. Reinbek bei Hamburg: Rowohlt.

Schönbächler, G., Stojkovic, D. \& Boothe, B. (2016): Mapping a gap: The concepts of the wish and wishing in psychoanalysis and the neurosciences, Neuropsychoanalysis, 18(2), 163-177.

Solms, M. (2018). Psychoanalyse und Naturwissenschaft. In F. Lackinger \& H. Rössler-Schülein (Hrsg.), Psychoanalyse und Universität. Zur Verbindung von akademischer Lehre und analytischer Praxis (S. 49-63). Giessen: Psychosozial-Verlag.

\section{Anmerkungen}

1 Das Gespräch fand Anfang März 2020 statt.

2 Anm. d. R.: In Zürich findet sich das Ludwik Fleck Zentrum für Wissenschaftstheorie als Forschungseinrichtung am Collegium Helveticum. Es verwaltet dessen Nachlass und fördert die Auseinandersetzung mit Flecks Denken. 